\title{
3 Research Square

\section{Elevated cellular PpIX potentiates sonodynamic therapy in a mouse glioma stem cell-bearing glioma model by downregulating the Akt/NFkB/MDR1 pathway}

Yoshifumi Mizobuchi ( $\nabla$ y.mizobuchi1203@gmail.com )

University of Tokushima

Kenji Shono

University of Tokushima

Izumi Yamaguchi

University of Tokushima

Kohei Nakajima

University of Tokushima

Yuri Fujiwara

University of Tokushima

Toshitaka Fujihara

University of Tokushima

Keiko Kitazato

University of Tokushima

Kazuhito Matsuzaki

University of Tokushima

Yoshihiro Uto

University of Tokushima

Oltea Ulsula

Keio University

Hideyuki Saya

Keio University

Yasushi Takagi

University of Tokushima

\section{Research Article}

Keywords: Glioblastoma (GBM), therapeutic resistance

Posted Date: December 10th, 2020 
DOI: https://doi.org/10.21203/rs.3.rs-112376/v1

License: (c) (1) This work is licensed under a Creative Commons Attribution 4.0 International License. Read Full License

Version of Record: A version of this preprint was published at Scientific Reports on July 23rd, 2021. See the published version at https://doi.org/10.1038/s41598-021-93896-0. 


\section{Abstract}

Glioblastoma (GBM) has high mortality rates because of extremely therapeutic resistance. During surgical resection for GBM, 5-aminolevulinic acid (5-ALA)-induced protoporphyrin IX (PpIX) fluorescence is conventionally applied to distinguish GBM. However, surgical intervention is insufficient for high invasive GBM. Sonodynamic therapy (SDT) is an emerging and promising approach combined with lowintensity ultrasonication (US) and PpIX as a sonosensitizer for cancer, whereas its efficacy is limited. Based on our previous study that down-regulation of multidrug resistant protein (MDR1) in GBM augmented anti-tumor effects of chemotherapy, we hypothesized that elevation of cellular PpIX levels by down-regulation of MDR1 enhances anti-tumor effects by SDT. In high invasive progeny cells from mouse glioma stem cells (GSCs) and a GSC-bearing mouse glioma model, we assessed the anti-tumor effects of SDT with a COX-2 inhibitor, celecoxib. Down-regulation of MDR1 by celecoxib increased cellular PpIX levels, as well as valspodar, a MDR1 inhibitor and augmented anti-tumor effects of SDT. MDR1 downregulation via Akt/NF-kB pathway by celecoxib was confirmed, using a NF-kB inhibitor, CAPÉ. Thus, elevation of cellar PpIX by down-regulation of MDR1 via Akt/NF-kB pathway may be crucial to potentiate the efficacy of SDT in a site-directed manner and provide a promising new therapeutic strategy for GBM.

\section{Introduction}

Glioblastoma (GBM) is the most common primary malignant brain tumor and has a high recurrence and mortality rates. ${ }^{1,2}$ Various current therapeutic approaches including surgery, chemotherapy, radiotherapy, and immunotherapy are clinically applied for treating GBMs. ${ }^{3,4}$ However, surgical intervention is insufficient for eradicating GBM because of its extremely high invasiveness. ${ }^{3}$ Chemotherapy and radiotherapy not only damage tumor cells but also partly harm normal cells. Furthermore, tumor cells acquire chemo-resistance during treatment, thus deterring GBM treatment. Though various approaches including neoadjuvant anti-PD-1 immunotherapy have been studied, ${ }^{5,6}$ novel therapeutic strategies are required to improve the prognosis of GBM patients.

Sonodynamic therapy (SDT) is a promising and emerging noninvasive approach for cancer treatment. SDT involves a combination of ultrasonication (US) via low-intensity ultrasound imaging and specialized chemical agents known as sonosensitizers including 5-aminolevulinic acid (5-ALA)-induced protoporphyrin IX (PpIX). ${ }^{7,8}$ After oral administration, 5-ALA can accumulate in GBM and surrounding infiltrating cancer cells such as glioma stem cells outside of the tumor bulk. Once formed, PpIX emits fluorescence at a peak wavelength of $635 \mathrm{~nm}$ after excitation with light near the Soret band peak around $410 \mathrm{~nm} .{ }^{9}$ Microbubbles generated during US implode and release markedly high energy and initiate the emission of sonoluminescence light, subsequently leading to the generation of reactive oxygen species (ROS). The anti-tumor effect of SDT is primarily attributed to the generated ROS, suggesting that mitochondrial ROS generation is promoted under the presence of PpIX upon SDT, ${ }^{10}$ resulting in severe damage to tumor cells through hydrodynamic shear forces and selectively destroying tumor cells. ${ }^{10,11}$ Currently, 5-ALA is commonly administered to visualize GBM during resection surgery. It is selectively 
taken up by tumor cells upon delivery and localizes to the mitochondria, being converted to PpIX. ${ }^{10}$, Because of its specificity and effectiveness towards highly invasive GBM cells, ${ }^{11}$ SDT might potentially be a novel strategy for glioma therapy. However, SDT monotherapy cannot completely eradicate tumors. Since the efficacy of SDT depends on cellular PpIX levels to induce intrinsic caspase-dependent apoptosis, $^{9-13}$ it may need to elevate the cellular PpIX level to promote the effects of SDT with new methods including high-intensity focused US. ${ }^{14}$

P-Glycoprotein, referred to as multidrug resistance receptor (MDR1), is a transmembrane glycoprotein functioning as an efflux pump and conferring multidrug resistance in brain tumors. ${ }^{15,16}$ Elsewhere, we reported high expression of MDR1 in GBM and demonstrated that down-regulation of MDR1 via Akt/NFkB pathways upon transfection of the Ad-DKK3 gene augmented the anti-tumor effects of temozolomide in GBM cells and in a GBM-xenograft model. ${ }^{17}$ We also reported that a selective cyclooxygenase-2 (COX2) inhibitor, celecoxib exerted anti-tumor effects associated with the down-regulation of Akt/NF-kB pathways in mouse glioma stem cells (GSCs) and GSCs-bearing glioma model. ${ }^{18,19}$ Based on these findings, we hypothesized that down-regulation of MDR1 by celecoxib via Akt/NF-kB pathways may promote the uptake of 5-ALA into GBM, thereby elevating cellular PpIX levels and enhance the anti-tumor effects of SDT.

This study shows that elevation of cellular PpIX through celecoxib-mediated MDR1 down-regulation potentiates anti-tumor effects of SDT in a mouse GSC-bearing malignant glioma model which is highly invasive and similar to GBM in many respects. ${ }^{20,21}$

\section{Results}

\section{Effects of SDT on GSC cell lines}

We first assessed the time course of PpIX fluorescence after treatment with 5-ALA in subconfluent U251 cells. Since the luminescence intensity peaked at $3 \mathrm{~h}$ after treatment with 5-ALA and was retained for $12 \mathrm{~h}$ (Fig. 1a), we decided to perform US (1 MHz, $2 \mathrm{~W} / \mathrm{cm}^{2}$ for $2 \mathrm{~min}$ ) at $3 \mathrm{~h}$ after treatment with $1 \mu \mathrm{M}$ 5-ALA, based on previous study. ${ }^{10,12}$ The effects of SDT in GSCs was assessed $24 \mathrm{~h}$ after US (Fig. 1b). Apoptosis-associated up-regulation of cleaved caspase (cCasp)- $9,-3$, and PARP but not cCasp-8 was significantly elevated in the SDT group (Fig. 1C), which was consistent with the significant reduction of cell viability at $24 \mathrm{~h}$ after SDT (Fig. 1d). These findings indicate the induction of cell death associated with intrinsic apoptosis upon SDT in GSCs.

\section{Anti-tumor effects of SDT in the mouse GSC-bearing glioma model}

We further examined the effects of SDT in the mouse GSC-bearing glioma model (Fig. 2a). SDT was performed $7 \mathrm{~d}$ after GSC injection. One day after SDT, we observed the up-regulation of cCasp-9, -3 and PARP but not cCasp-8 on immunohistochemistry (Fig.2b), indicating the induction of intrinsic apoptosis by SDT in consistent with our in vitro findings (Fig. 1c). SDT significantly decreased the tumor size (Fig. 
2c) without affecting body weights $14 \mathrm{~d}$ after GSC injection (Fig. 2d) but did not significantly extend the survival period, compared to the other groups (Fig. 2e). The anti-tumor effects of single SDT may be limited in this model.

\section{An increase in cellular PpIX via celecoxib-mediated MDR1 down-regulation augmented the anti-tumor effect of SDT}

To enhance the anti-tumor effects of SDT, we examined whether MDR1 down-regulation can increase the cellular PpIX levels after 5-ALA injection. Based on our previous findings in human glioma cells, ${ }^{17}$ we first observed that the expression levels of COX-2 and MDR1 in human glioblastoma and the mouse GSCbearing glioma model were higher than those in normal brain tissue (Fig. 3a). Using a COX-2 inhibitor, celecoxib, we assessed the effects on the MDR1 expression in GSCs. Treatment with celecoxib $60 \mu \mathrm{M}$ (IC ${ }_{50}$ dose) decreased MDR1 expression levels and the PpIX levels were significantly higher upon combination treatment with $1 \mu \mathrm{M}$ 5-ALA and celecoxib than with 5-ALA monotherapy (Fig. 3b). To confirm the MDR1-dependent regulation of PpIX levels, we examined the effects of an MDR1 inhibitor, valspodar (Fig. 3c). MDR1 was down-regulated by $1 \mu \mathrm{M}$ valspodar (Fig. 3c) and cellular PpIX levels increased as expected. Concurrent with these findings, combination therapy with SDT and valspodar significantly decreased cell viability compared to each single treatment (Fig. 3d). The increase in cellular PpIX levels through MDR1 down-regulation may be at least partly attributable to the enhancement of the anti-tumor effects of SDT.

\section{Combination therapy with SDT and celecoxib enhanced apoptosis induction, thereby enhancing anti- tumor effects in the mouse GSC-bearing glioma model}

The anti-tumor effects by combination of SDT with celecoxib was assessed in the mouse GSC-bearing glioma model (Fig. 4a). Compared to normal tissue, the expression of MDR1 was elevated in brain tumor

tissue after GSCs injection, which was reduced upon treatment with $10 \mathrm{mg} / \mathrm{kg}$ celecoxib for 7 days before SDT (Fig. 4b). Concurrent with this finding, cellular PpIX levels in brain tumor after 5-ALA injection were significantly increased (Fig. 4b). The elevation of cellular PpIX levels upon combination therapy with SDT and celecoxib escalated the induction of intrinsic and extrinsic apoptosis 1 day after SDT (Fig. 4c), thus decreasing the tumor volume (Fig 4d) and prolonging survival (Fig. 4e). The combination therapy with SDT and celecoxib may be a promising method for GBM treatment.

\section{Enhancement of anti-tumor effects by SDT was attributed to celecoxib-mediated MDR1 down-regulation through the AKT/NF-KB pathway}

To clarify the mechanisms underlying celecoxib-mediated MDR1 down-regulation, we treated (i.p.) the mouse GSC-bearing glioma model with $10 \mathrm{mg} / \mathrm{kg}$ celecoxib for 14 days (Fig. 5a). AKT2, pAkt, pNF-kB, and MDR1 were significantly up-regulated in the glioma model than in normal tissue and were attenuated through celecoxib treatment (Fig. 5a). Furthermore, to confirm the transcriptional regulation of MDR1, we used a proteasome inhibitor, caffeic acid phenethyl ester (CAPE), which inhibits the phosphorylation of inhibitor of $\mathrm{kB}(\mathrm{I}-\mathrm{kB})$, thus facilitating the phosphorylation of NF-kB (pNF-kB) and regulating the 
transcription of target genes. In GSCs, up-regulation of MDR1 mRNA and protein was significantly attenuated upon treatment with $1 \mu \mathrm{M}$ CAPE, accompanied by the reduction of pl-kB and pNF-kB (Fig. 5b), suggesting transcriptional regulation of MDR1 through NF-kB. Together, celecoxib-mediated MDR1 downregulation through the Akt/NF- kB pathway may increase cellular PpIX levels through increased intratumoral uptake of 5-ALA, thus promoting apoptosis induction via SDT (Fig. 5C). MDR1 downregulation may be attributable to the improvement of not only chemo-resistance, but also the therapeutic efficacy of SDT in GBM.

\section{Discussion}

In this study, we initially show that combination of SDT and celecoxib to down-regulate MDR1 augmented the anti-tumor effects in GSCs and the mouse GSC-bearing malignant glioma model indicating invasive and therapeutic resistance. First, we confirmed that SDT monotherapy exerted antitumor effects by inducing intrinsic apoptosis in GSCs and GSCs-bearing glioma model; however, it displayed transient and limited efficacy. Since we have demonstrated the anti-tumor effects by a COX2 inhibitor, celecoxib, ${ }^{19}$ we assessed the efficacy of SDT combined with pre-treatment by celecoxib. Expectedly, the pre-treatment by celecoxib decreased the expression of MDR-1 and elevated the cellular PpIX levels induced by 5-ALA, resulting the enhanced anti-tumor effects of the combination therapy. Next, to confirm the mechanisms underlying the downregulation of MDR-1 by celecoxib, we used a MDR1inhibitor, valspodar. It decreased the expression of MDR-1 and the cell-viability. Finally, we verified the down-regulation of MDR-1 by celecoxib via Akt/NF-kB pathway, using CAPE, a NF-kB inhibitor. The combination therapy of SDT and celecoxib enhanced the induction of both intrinsic and extrinsic apoptosis pathways in the GSCs-bearing malignant glioma model. Taken together, combination therapy with SDT and celecoxib may be a promising strategy to retard GBM recurrence.

SDT has been developed as a novel promising noninvasive approach derived from photodynamic therapy

(PDT). ${ }^{22,23}$ Because PDT is tightly focused with a short penetration depth of light in soft tissue up to several tens of centimeters, PDT is not effective for the treatment of deep-seated tumors. ${ }^{24,}{ }^{25}$ In contrast, SDT is anticipated to overcome the major limitation of PDT. It may be a promising treatment method for brain tumors and other tumors. ${ }^{26} \mathrm{PpIX}$ converted from 5-ALA in mitochondria acts to form heme by the enzyme ferrochelatase. In GBM, the decrease of ferrochelatase may contribute to the accumulation of PpIX within gliomas and GSCs and lead to its preferential localization in tumor. ${ }^{9}$ Although we did not assess the effects of ferrochelatase, we suggested that the increased cellular PpIX level may be at least partly attributable to the uptake of 5-ALA into brain or GSCs through down-regulation of MDR-1 by celecoxib, thereby enhancing anti-tumor effects. Because GBM occurs in the brain parenchyma surrounded by the skull and differs from other epithelial cancers, thus far, SDT is performed for GBM patients only during surgical intervention. ${ }^{27}$ Although the ultrasound instrument to make possible the repeat application of SDT had developed, the applicable institutes are limited. Therefore, to enhance the repeatability of SDT, the development of feasible and safe equipment with new sonosensitizers ${ }^{28}$ are urgently required. 
MDR1 (ABCB1) gene encodes P-glycoprotein, a drug transporter that is a critical component of the bloodbrain barrier, which prevents entry of many potentially toxic compounds into the central nervous system. ${ }^{29}$ We previously reported that the reduction of MDR1 upon transfection of DKK3 gene in human glioma cells enhanced the anti-tumor effects of temozolomide. ${ }^{17}$ Concurrent with previous study indicated the increased mRNA levels of MDR1 in COX-2-overexpressing cells, the present study showed that MDR1 was highly up-regulated and COX-2 was overexpressed in GSC-derived glioma tissue and in human glioma tissue. COX-2, an inducible form of the enzyme catalyzing the first step in prostanoid synthesis, is reported and is overexpressed in various tumors and possesses proangiogenic and anti-apoptotic properties. Thus, up-regulation of MDR1and COX-2 may be associated with not only chemotherapeutic resistance but also with the limited uptake of 5-ALA in GSCs and the mouse GSC-bearing glioma model. To enhance the efficacy of SDT, we assessed the cellular up-take of 5-ALA upon MDR1 down-regulation in GSCs treated with celecoxib and an MDR1 inhibitor, valspodar. As expected, cellular PpIX levels increased upon MDR1 down-regulation. MDR1 is expressed in normal liver and kidney tissue, where it functions to actively transport lipophilic xenobiotic compounds and serves as an efflux pump for chemotherapeutic agents. ${ }^{30}$ Therefore, MDR1 down-regulation by a COX-2 inhibitor might be essential for increasing the intratumoral uptake of anti-tumor drugs and 5-ALA. In addition, MDR1 expression is associated with several cellular signaling pathways and protein kinases, chaperons, ubiquitin-related enzymes, and transcription factors. ${ }^{31}$ As the COX-2 inhibitor induced apoptosis by inhibiting the AKT pathway in lowgrade glioma cells in a previous study, ${ }^{18}$ we observed MDR1 down-regulation through the Akt/NF-kB pathway upon celecoxib treatment. Furthermore, we observed the down-regulation of MDR1 by a NF-kB inhibitor, CAPE. Thus, celecoxib may affect both apoptosis induction and MDR1 down-regulation by inhibiting the Akt/NF-kB pathway, resulting in an enhanced anti-tumor effects of not only SDT but also other anti-tumor agents.

This study has some limitations. Within a short period after SDT, apoptosis induction was enhanced in combination with celecoxib, and the cell viability was decreased. Therefore, we need the assessment of anti-tumor effects through repeated SDT upon combination during a longer period. treatment with celecoxib. We previously confirmed that on combination treatment with 5-ALA and US, ROS generation and anti-tumor effects were greater than those upon monotherapy. ${ }^{10}$ Unfortunately, in the current study, we did not assess the ROS generation, whereas we confirmed the elevated cellular PpIX level. Then we need to confirm whether elevated PpIX in combination therapy with SDT and celecoxib is associated with ROS generation, thereby leading to the enhancement of anti-tumor effects by combination therapy with SDT and celecoxib on human glioma stem cells. SDT activates the mitochondrial caspase pathway and down-regulates ATP-binding cassette transporters such as MDR1, thus selectively improving the uptake of chemotherapeutic drugs into tumor cells and reducing the toxic effects on normal cells and tissues. 31,32 Therefore, sequential treatment with celecoxib after SDT may improve the uptake of celecoxib by itself into glioma cells, contributing to the prolonged survival in our glioma model. Although combination therapy with SDT and celecoxib may exert various synergistic therapeutic effects on GBM, we cannot exclude the favorable effects of celecoxib after SDT beyond MDR1 down-regulation. ${ }^{33}$ Further studies are required to develop clinically applicable, handy, repeatable, and feasible devices for SDT. 
In conclusion, the combination therapy with SDT and celecoxib resulted in enhanced anti-tumor efficacy among GSCs and a mouse GSC-bearing glioma model. MDR1 down-regulation via the Akt/NF-kB pathway may be a promising mean for treatment of GBM patients. Our results warrant for further verification of the anti-tumor effects of combination therapy with SDT and other chemotherapeutic agents and the development of new US systems capable of penetrating the skull.

\section{Methods}

\section{Study approval and informed consent}

This study was approved by the ethical review board of Tokushima University Hospital for human study and the ethics committee of Tokushima University Graduate School of Biomedical Sciences, Tokushima, Japan. Human tissue samples were obtained during routine clinical procedures after informed consent including the use of tissue sample from patients with brain tumors at the Department of Neurosurgery, Tokushima University Hospital. For the use of samples obtained from patients, we have obtained a statement attesting to informed consent for all patients with or without neurosurgery in our department. Each sample was fixed in $4 \%$ formalin in phosphate-buffered saline (PBS) and processed for paraffin embedding. The samples were classified by neuropathologists in accordance with the WHO classification of brain tumors. Sections from non-neoplastic regions (NNRs) were purchased from BioChain Institute (Newark, NJ, USA). The study was performed in accordance with the tenets of the Declaration of Helsinki. All animal experiments were approved and performed in accordance with the animal care guidelines of Tokushima University.

\section{Cell Lines}

Human GBM cell line U251MG were purchased from American Type Culture Collection (Manassas, VA, USA) and cultured in RPMI-1640 medium (Invitrogen, NJ, USA) with 10\% fetal bovine serum (GIBCO-BRL, NY, USA) at $37^{\circ} \mathrm{C}$ in an atmosphere of $5 \% \mathrm{CO}_{2}$ and $95 \%$ humidified air. Mice GSCs were established and provided by OU and HS, Keio University. ${ }^{19,20}$ GSCs were cultured in Dulbecco's Modified Eagle's medium/nutrient mixture F-12 Ham (Sigma-Aldrich, St. Louis, MO, USA) supplemented with $20 \mathrm{ng} / \mathrm{ml}$ recombinant human epidermal growth factor (PeproTech, Rocky Hill, NJ, USA), $20 \mathrm{ng} / \mathrm{ml}$ recombinant human basic fibroblast growth factor (PeproTech), B-27 supplement without vitamin A (Life Technologies, Carlsbad, CA, USA), $200 \mathrm{ng} / \mathrm{ml}$ heparin sulfate, $100 \mathrm{U} / \mathrm{ml}$ penicillin, and $100 \mu \mathrm{g} / \mathrm{ml}$ streptomycin (Nacalai Tesque, Kyoto, Japan).

\section{Cell Viability Assay}

GSCs $\left(1 \times 10^{3}\right.$ cells/well) were plated in 96 -well tissue culture plates. To enumerate viable cells, the conversion of WST-8 to formazan by metabolically active cells was quantified using WST-8 reagent (Dojindo, Osaka, Japan) on a microplate reader (Infinit F200 PRO ${ }^{\circledR}$, TECAN) at $450 \mathrm{~nm}$. We used PBStreated cells as the control to represent $100 \%$ viability and the percent viability was determined in each treatment. 


\section{Establishment of the animal model and assessment of anti-tumor effects}

Six-week-old male C57BL/6 mice were subjected to inhalation anesthesia with isoflurane and a stereotactic apparatus was placed in the right brain. With a dental drill, a small hole was bored into the skull $2.0 \mathrm{~mm}$ lateral to the bregma. Using a malignant glioma model with mouse GSCs established by Sampetrean, $\mathrm{O}$ and Saya, $\mathrm{H}$ et al, ${ }^{20,21} \mathrm{GSC}$ progeny cells $\left(1 \times 10^{3}\right)$ in $2 \mu \mathrm{l}$ of Hank's balanced salt solution (Sigma-Aldrich) were injected into the right cerebral hemisphere $3 \mathrm{~mm}$ below the brain surface, using a 10- $\mu$ l Hamilton syringe. To examine the anti-tumor effects of SDT, the mice were randomized and treated with 5-ALA, US, or SDT and compared to the non-treated control (Figure 2). For SDT, a 0.2-ml solution of 5 -ALA in PBS was intraperitoneally injected at a dose of $200 \mathrm{mg} / \mathrm{kg}$ body weight. Three hours later, the mouse right brain was placed on the stereotactic apparatus and subjected to US imaging $(1 \mathrm{MHz}, 2$ $\mathrm{W} / \mathrm{cm}^{2}$ for $2 \mathrm{~min}$ ) under inhalation anesthesia. On day 1 after SDT, apoptosis induction by SDT was confirmed and tumor volume on days 3, 7, and 14 and the survival rate were analyzed. Mice were euthanized and their brains were sliced on a brain slicer matrix at 1.0-mm intervals and the tumor volume, represented by the GFP-positive area, was microscopically determined (Keyence BZ-X710, Osaka, Japan).

In addition, to assess the effect of combination therapy of SDT and celecoxib, another set of mice were randomized and treated with vehicle, celecoxib, SDT, or a combination of celecoxib and SDT (Figure 4). Celecoxib, lysed with dimethyl sulfoxide (DMSO) and hydroxypropyl- $\beta$-cyclodextrin (HBC), was injected (i.p.) at a dose of $10 \mathrm{mg} / \mathrm{kg}$ consecutively after mouse GSC implantation. Vehicle controls received equivalent doses of DMSO/HBC and normal saline at the same dosing schedule. To validate the efficacy of celecoxib during SDT, tumor volume on day 14 and the survival rate during the observation period were assessed in each group (Figure 4) as described above.

\section{Measurement of cellular PpIX levels and SDT}

Luminescence was measured $1,2,3,6$, and $12 \mathrm{~h}$ after treatment of human GBM U251 cells with $1 \mu \mathrm{M} 5$ ALA in human GBM U251 cells, using the image analyzer in the BZ-X710 microscope (KEYENCE). Thereafter, the viability of GSCs upon combination therapy with SDT and US ( $1 \mathrm{MHz}, 2 \mathrm{~W} / \mathrm{cm}^{2}$ for 2 min)at $3 \mathrm{~h}$ after 5-ALA treatment was assessed after $24 \mathrm{~h}$.

Celecoxib (Sigma-Aldrich, Cat. \#PHR1683) was dissolved in DMSO and supplemented in the culture medium at a final concentration of $60 \mu \mathrm{M}$. After 3-h incubation of cells in medium supplemented with 1 $\mu \mathrm{M}$ 5-ALA (SBI ALA promo, Tokyo, Japan), the medium was replaced with fresh complete medium, and the 96 -well plate was exposed to LED irradiation $\left(630 \mathrm{~nm}, 80 \mathrm{~mW} / \mathrm{cm}^{2}\right)$ for $5 \mathrm{~min}$. The LED light spot was an equally illuminated rectangular spot encompassing the entire culture plate. SDT was performed using the ultrasonic generator UST-770 (ITO Co. Ltd., Tokyo, Japan). In the mouse GSC-bearing glioma model, the tumor area was extracted $3 \mathrm{~h}$ after treatment with $200 \mathrm{mg} / \mathrm{kg} 5-\mathrm{ALA}$ and PpIX levels were analyzed as previously described. ${ }^{20}$ 
Total RNA was isolated and extracted using the MagNA Pure RNA isolation kit (Roche, Tokyo, Japan) and the MagNa lyser (Roche), in accordance with the manufacturer's instructions. We used Transcriptor Universal cDNA Master (Roche) to reverse-transcribe total RNA to cDNA and a LightCycler 2.0 (Roche Diagnostics, Tokyo, Japan) for qRT-PCR. The following primers were used: mouse mdr1, 5'-primer, GGC ATT GCC TAC CTG TTG G-3'; 3'-primer GCT TTC TGT GGA CAC TTC TG, and mouse glyceraldehyde-3phosphate dehydrogenase (Gapdh), 5'-CAG AAC ATC ATC CCT GCA TC-3' and 5'-CTG CTT CAC CAC CTT CTT GA-3'. The mRNA levels were normalized to those of Gapdh. The PCR conditions were as follows: $95^{\circ} \mathrm{C}$ for $10 \mathrm{~min}$, followed by 40 cycles at $95^{\circ} \mathrm{C}$ for $10 \mathrm{~s}, 60^{\circ} \mathrm{C}$ for $10 \mathrm{~s}$, and $72^{\circ} \mathrm{C}$ for $8 \mathrm{~s}$. We subjected 4 samples in each group to the qRT-PCR assay to determine the gene expression levels.

\section{Western blot analysis}

According to our previous study (6), cells or tissue samples were homogenized in RIPA buffer containing a protease/phosphatase inhibitor cocktail (Cell Signaling Technology, Cat. \#5872). After 10-min centrifugation at $12000 \mathrm{rpm}, 4^{\circ} \mathrm{C}$, the protein concentration in the supernatants was determined using BCA kit (Thermo Fisher Scientific, USA). Protein ( 20 or $50 \mathrm{mg}$ ) was separated by SDS-PAGE and transferred to polyvinylidene fluoride membranes (immune-blot PVDF membrane, BIO-RAD, Hercules, CA, USA) by electroblotting. The membrane were immersed in blocking buffer ( $5 \%$ skim milk or $2 \%$ BSA in trisbuffered saline, TBS) for $1 \mathrm{~h}$ and incubated with primary antibodies: anti-MDR1 (BD Biosciences, NJ, USA, 1:1000), anti-cCaspase-8, $-9,-3$ and anti-PARP (Cell Signaling Technology, MA, USA, 1:1000), antiAKT2 (Abcam, Cambridge, UK, rabbit, 1:1000), pAkt (Santa Cruz Biotechnology, CA, USA, rabbit, 1:500), anti-pNF- kB (Cell Signaling Technology, 1:1000), anti-pl-kB (Cell Signaling Technology, 1:1000) and $\beta$-actin (Sigma-Aldrich, mouse, 1:5000) were diluted in Can Get Signal Solution 1 (Toyobo). After washing in Tween-TBS (T-TBS), the membranes were incubated for $1 \mathrm{~h}$ with horseradish peroxidaseconjugated secondary antibodies in Can Get Signal Solution 2 (dilution 1:3000). After washing, the protein-antibody complexes were detected with Amersham ECL prime Western blotting detection reagents (GE Healthcare, UK) using a Lumino image analyzer (Image Quant LAS-4000 mini, GE Healthcare Japan, Tokyo, Japan) and ImageJ 1.52 software (NIH, Bethesda, MD, USA) was used to analyze the protein expression levels. Each experiment was repeated four times.

\section{Immunohistochemistry}

Murine tissue samples were fixed with 4\% paraformaldehyde and 5- $\mu$ m-thick frozen sections were mounted on Matsunami adhesive saline (MAS)-coated glass slides (Matsunami Glass, Tokyo, Japan). As previously reported (6), human glioma tissue sections from the paraffin-embedded block were dewaxed, rehydrated, and subjected to antigen retrieval. The sections were blocked for $30 \mathrm{~min}$ with $1-3 \%$ hydrogen peroxide solution, and stained overnight at $4{ }^{\circ} \mathrm{C}$ with the following antibodies: anti-MDR1 (D-11) (Santa Cruz Biotechnology, Inc., Dallas, TX, USA, 1:100), anti-COX-2 (Abcam, Cat. \# ab15191), rabbit monoclonal anti-MDR1 (Abcam; ab170904; 1:100), anti-cleaved caspase-8 (cCasp-8), anti-cCasp-9, anti-cCasp-3, and anti-PARP (Cell Signaling Technology, 1:1000). Thereafter, they were incubated with biotinylated 
secondary antibody $\left(30 \mathrm{~min}, 30^{\circ} \mathrm{C}\right)$, visualized using DAB buffer tablets, and counterstained with hematoxylin. Photographs were obtained under a light microscope, using KEYENCE BZ-X710.

\section{Statistical analysis}

Survival estimates and median survivals were determined using Kaplan-Meier survival curves. A log-rank (Mantel-Cox) test was performed to determine the p-values derived from Kaplan-Meier survival curves. To determine statistical significance, between-group comparisons were performed using Student's $t$-test. For multiple comparisons, one-way ANOVA, followed by the Tukey-Kramer tests. Error bars indicate the standard deviation values. All statistical analyses were performed using JMP 13.2 (SAS Institute Inc.) and the differences with $p<0.05$ were considered significant.

\section{Declarations}

\section{Competing interests}

We have no conflict of interest.

\section{Acknowledgements}

We thank Emiko Nishikawa and Akiko Sumi for excellent technical support.

\section{Funding}

Funding for this work was provided by a Grant-in-Aid for Scientific Research (No. 18K16586 to K.S.) and (No. 25462264 to Y. M.) from the Ministry of Education, Culture, Sports, Science, and Technology of Japan.

\section{Authors' contributions}

$\mathrm{KS}$ and IY performed the in vivo and in vitro experiments; TF, KN, YF and KM performed the in vitro experiments; OU and HS provided transgenic cell; $\mathrm{YU}$ provided ultrasonic generator; $\mathrm{YM}$ and KTK designed the work and contributed at the revision stage of the paper; YT designed and organized the work; all of the authors contributed to the final version of the paper.

\section{References}

1. Ostrom, Q. T., Bauchet, L., Davis, F. G., Deltour, I., Fisher, J. L., Langer, C. E., Pekmezci, M., Schwartzbaum, J. A., Turner, M. C., Walsh, K. M., Wrensch, M. R., Barnholtz-Sloan, J. S.The Epidemiology of Glioma in Adults: A "State of the Science" Review. Neuro Oncol. 17, 624-626 (2015).

2. Lu, V. M., Jue, T. R., McDonald, K. L., Rovin, R. A. The Survival, Effect of Repeat Surgery at Glioblastoma Recurrence and its Trend: A Systematic Review and Meta-Analysis. World Neurosurg. 115, 453-459 (2018). 
3. Hervey-Jumper, S. L., Berger, M. S. Maximizing safe resection of low- and high-grade glioma. Neurooncol. 130, 269-282 (2016).

4. Bush N. A., Chang, S. M., Berger, M. S. Current and future strategies for treatment of glioma. Rev.40, 1-14 (2017).

5. Adhikaree, J., Moreno-Vicente, J., Kaur, A. P., Jackson, A. M., Patel, P. M. Resistance Mechanisms and Barriers to Successful Immunotherapy for Treating Glioblastoma. Cells 21, 263 (2020).

6. Yamaguchi, I., Nakajima, K., Shono, K., Mizobuchi, Y., Fujihara, T., Shikata, E., Yamaguchi, T., Kitazato, K., Sampetrean, O., Saya, H., Takagi, Y. Downregulation of PD-L1 via FKBP5 by celecoxib augments antitumor effects of PD-1 blockade in a malignant glioma model. Neuro-Oncol. Adv. 2, vdz058 (2020).

7. Wu, S. K., Santos, M. A., Marcus, S. L. Hynynen, K. MR-guided Focused Ultrasound Facilitates Sonodynamic Therapy with 5-Aminolevulinic Acid in a Rat Glioma Model. Sci Rep. 9, 1-10 (2019).

8. Mason, T. J. Therapeutic ultrasound an overview. Sonochem, 18, 847-852 (2011).

9. Mahmoudi K, Garvey KL, Bouras A, Cramer G, Stepp H, Jesu Raj JG, Bozec D, Busch TM, Hadjipanayis CG. 5-aminolevulinic acid photodynamic therapy for the treatment of high-grade gliomas. J Neurooncol. 141:595-607 (2019).

10. Shimamura, Y., Tamatani, D., Kuniyasu, S., Mizuki, Y., Suzuki, T., Katsura, H., Yamada, H., Endo, Y., Osaki, T., Ishizuka, M., Tanaka, T., Yamanaka, N., Kurahashi, T., Uto, Y. 5-Aminolevulinic acid enhances ultrasound-mediated antitumor activity via mitochondrial oxidative damage in breast cancer. Anticancer Res. 36, 3607-3612 (2016).

11. Prada, F., Kalani, M. Y. S., Yagmurlu, K., Norat, P., Del Bene, M., DiMeco, F., Kassell, N. F. Applications of Focused Ultrasound in Cerebrovascular Diseases and Brain Tumors. Neurotherapeutics 16, 67-87 (2019).

12. Song, D., Yue, W., Li, Z., Li, J., Zhao, J., Zhang, N. Study of the mechanism of sonodynamic therapy in a rat glioma model. OncoTargets Ther. 7, 1801-1810 (2014).

13. Chen, Z., Li, J., Song, X., Wang, Z., Yue, W. Use of a novel sonosensitizer in sonodynamic therapy of U251 glioma cells in vitro. Exp. Ther. Med. 3, 273-278 (2012).

14. Suehiro, S., Ohnishi, T., Yamashita, D., Kohno, S., Inoue, A., Nishikawa, M., Ohue, S., Tanaka, J., Kunieda, T. Enhancement of antitumor activity by using 5-ALA-mediated sonodynamic therapy to induce apoptosis in malignant gliomas: significance of high-intensity focused ultrasound on 5-ALASDT in a mouse glioma model. J. Neurosurg. 129, 1416-1428 (2018).

15. Chen, L., Shi, L., Wang, W., Zhou, Y. ABCG2 downregulation in glioma stem cells enhances the therapeutic efficacy of demethoxycurcumin. Oncotarget 8, 43237-43247 (2017).

16. de Trizio, I., Errede, M., d'Amati, A., Girolamo, F., Virgintino, D. Expression of P-gp in Glioblastoma: What We Can Learn From Brain Development. Curr. Pharm. Des. 26, 1428-1437 (2020).

17. Fujihara, T., Mizobuchi, Y., Nakajima, K., Kageji, T., Matsuzaki, K., Kitazato, K. T., Otsuka, R., Hara, K., Mure, H., Okazaki, T., Kuwayama, K., Nagahiro, S., Takagi, Y. Down-regulation of MDR1 by Ad-DKK3 
via Akt/NFkB pathways augments the anti-tumor effect of temozolomide in glioblastoma cells and a murine xenograft model. J. Neurooncol. 139, 323-332 (2018).

18. Shono K, Yamaguchi I, Mozobuchi Y, Takagi Y et al. Downregulation of the CCL2/CCR2 and CXCL10/CXCR3 axes contributes to antitumor effects in a mouse model of malignant glioma. Sci Rep. 17;10(1):15286 (2020).

19. Sato, A., Mizobuchi, Y., Nakajima, K., Shono, K., Fujihara, T., Kageji, T., Kitazato, K., Matsuzaki, K., Mure, H., Kuwayama, K., Sumi, A., Saya, H., Sampetrean, O., Nagahirao, S. Blocking COX-2 induces apoptosis and inhibits cell proliferation via the Akt/survivin- and Akt/ID3 pathway in low-gradeglioma. Neurooncol. 132, 231-238 (2017).

20. Sampetrean, O., Saya, H. Characteristics of glioma stem cells. Brain Tumor Pathol. 30, 209-214 (2013).

21. Shibao, S., Minami, N., Koike, N., Fukui, N., Yoshida, K., Saya, H., Sampetrean, O. Metabolic heterogeneity and plasticity of glioma stem cells in a mouse glioblastoma model. Neuro-Oncology 20, 343-354 (2018).

22. Shinohara, Y., et al. Development of a novel Schiff base derivative for enhancing the anticancer potential of 5-aminolevulinic acid-based photodynamic therapy. Photodiagnosis Photodyn. Ther. 20, 182-188 (2017).

23. Bilmin, K., Kujawska, T., Grieb, P. Sonodynamic Therapy for Gliomas. Perspectives and Prospects of Selective Sonosensitization of Glioma Cells. Cells 8, 1428 (2019).

24. Stepp, H., Stummer, W. 5-ALA in the management of malignant glioma. Lasers Surg. Med. 50, 399419 (2018).

25. Dolmans, D. E., Fukumura, D., Jain, R. K. Photodynamic therapy for cancer. Rev. Cancer 3, 380-387 (2003).

26. Yuan, S. X., et al. Underlying mechanism of the photodynamic activity of hematoporphyrin-induced apoptosis in U87 glioma cells. J. Mol. Med. 41, 2288-2296 (2018).

27. Sun, Y., Wang, H., Wang, P., Zhang, K., Geng, X., Liu, Q., Wang, X. Tumor targeting DVDMSnanoliposomes for an enhanced sonodynamic therapy of gliomas. Sci. 26, 985-994 (2019).

28. Bilmin, K., Kujawska, T., Grieb, P. Sonodynamic Therapy for Gliomas. Perspectives and Prospects of Selective Sonosensitization of Glioma Cells. Cells 13, 1428 (2019).

29. Munoz, J. L., Walker, N. D., Scotto, K. W., Rameshwar, P. Temozolomide competes for P-glycoprotein and contributes to chemoresistance in glioblastoma cells. Cancer Lett. 10, 69-75 (2015).

30. Patel, V. A., Dunn, M. J., Sorokin, A. Regulation of MDR-1 (P-glycoprotein) by cyclooxygenase-2. Biol. Chem. 277, 38915-38920 (2002).

31. Robey, R. W., Pluchino, K. M., Hall, M. D., Fojo, A. T., Bates, S. E., Gottesman, M. M. Revisiting the role of $A B C$ transporters in multidrug-resistant cancer. Rev. Cancer. 18, 452-464 (2018).

32. Xi, G., et al. CD133 and DNA-PK regulate MDR1via the PI3K- or Akt-NF-KB pathway in multidrugresistant glioblastoma cells in vitro. Oncogene 35, 241-250 (2016). 
33. Lin, F., et al. PI3K-mTOR Pathway Inhibition Exhibits Efficacy Against High-grade Gliomain Clinically Relevant Mouse Models. Cancer Res. 23, 1286-1298 (2017).

\section{Figures}

a. In U251 $1 \times 10^{6}$ human GBM cells
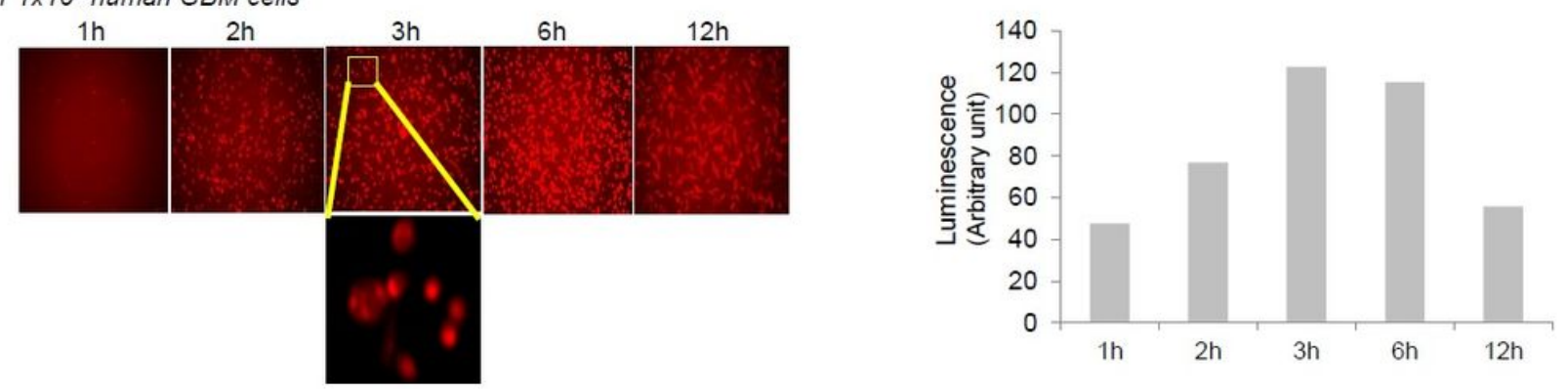

b.
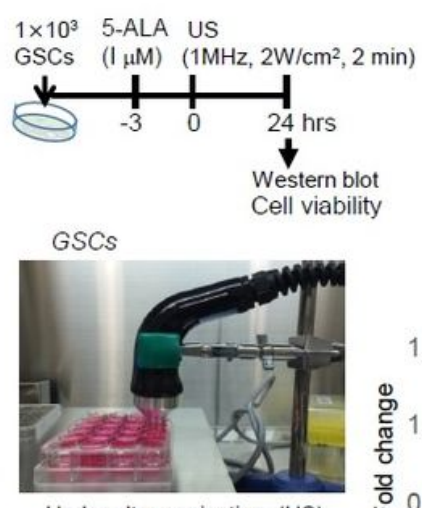

Under ultra sonication (US) c.
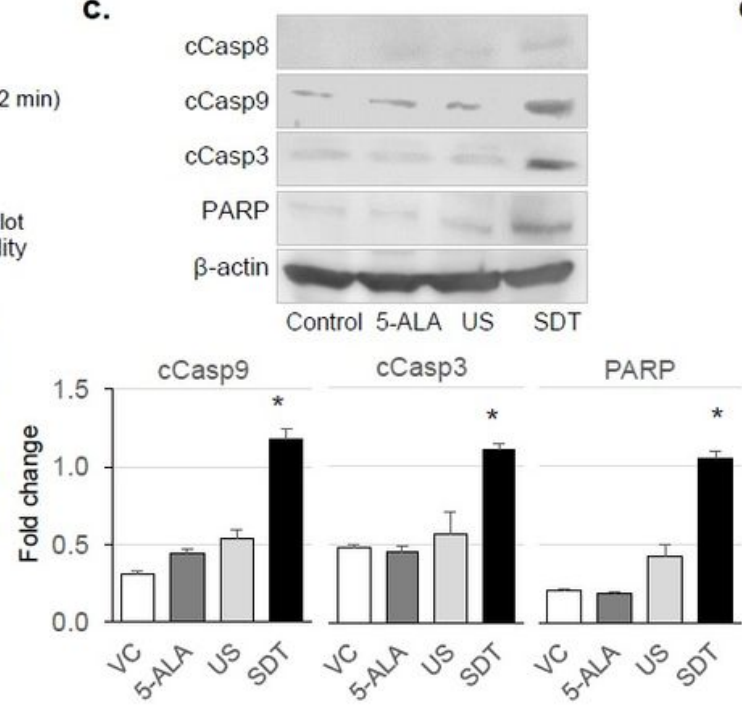

d.

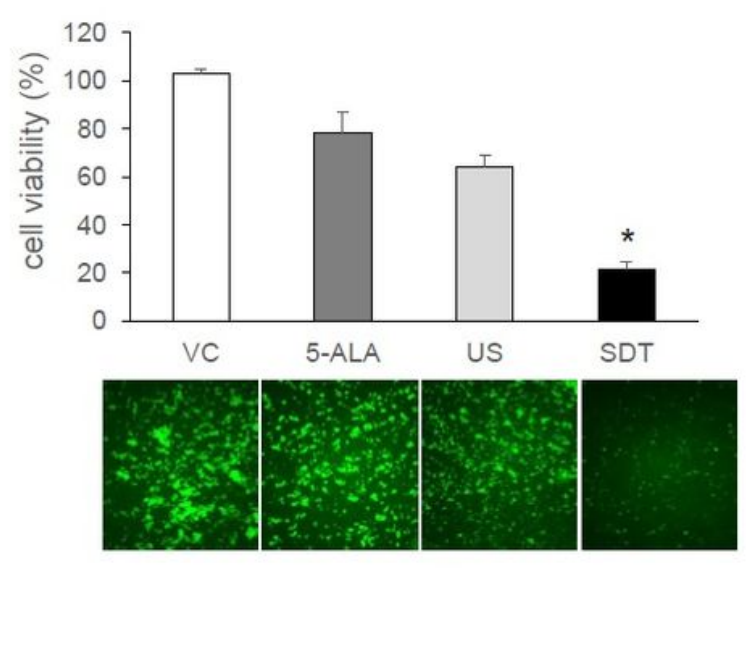

\section{Figure 1}

Cell viability by sonodynamic therapy (SDT) in mouse glioma stem cells (GSC). a. Changes of luminescence images and intensity in human glioblastoma $\mathrm{U} 251$ cells for $12 \mathrm{~h}$ after treatment with $1 \mu \mathrm{M}$ 5-aminolevulinic acid (5-ALA) as a sonosensitizer b. Protocol and photos under ultrasonication (US) c. Western blotting (WB) analysis of apoptosis induced by sonodynamic therapy (SDT). d. Cell viability by SDT; a combination of low-intensity ultrasonication (US) $3 \mathrm{~h}$ after treatment with $1 \mu \mathrm{M} 5$-ALA. ${ }^{*} \mathrm{p}<0.05$ by Turkey-Kramer vs others 
a.
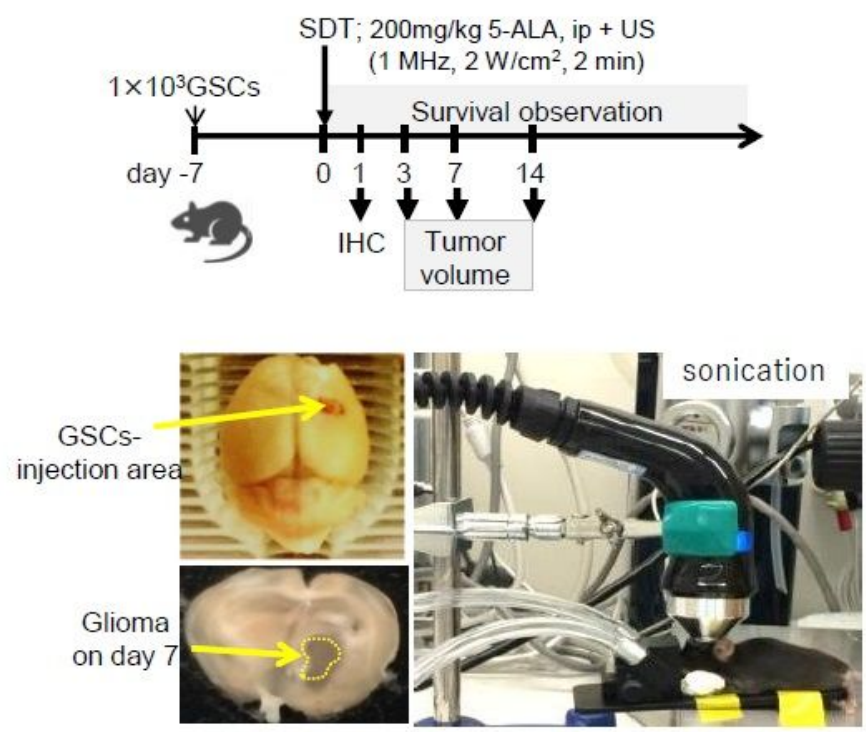

d.

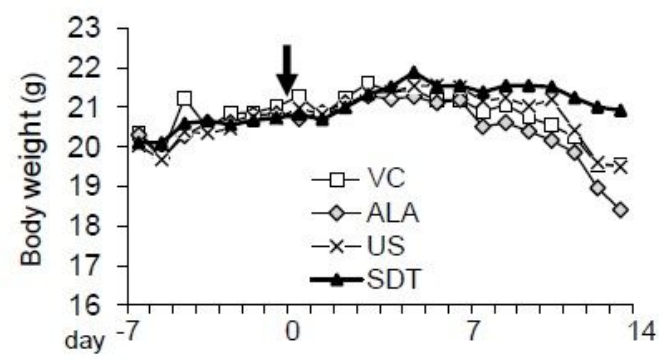

b.

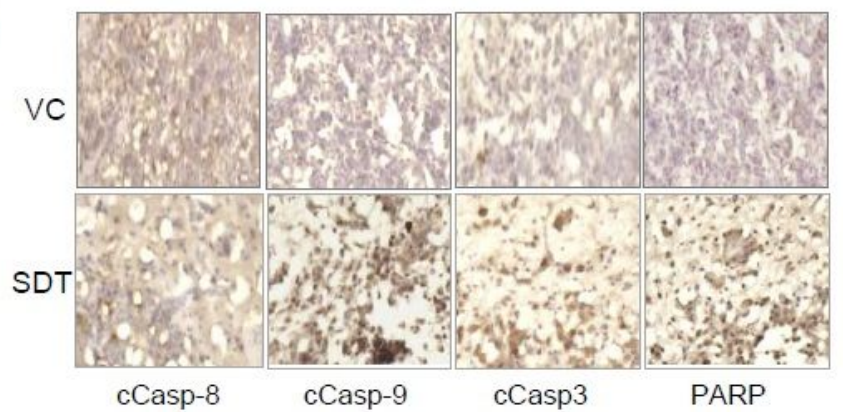

c.
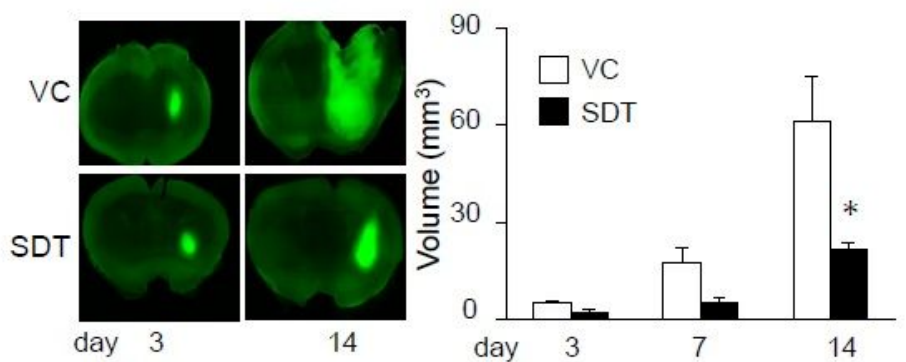

e.

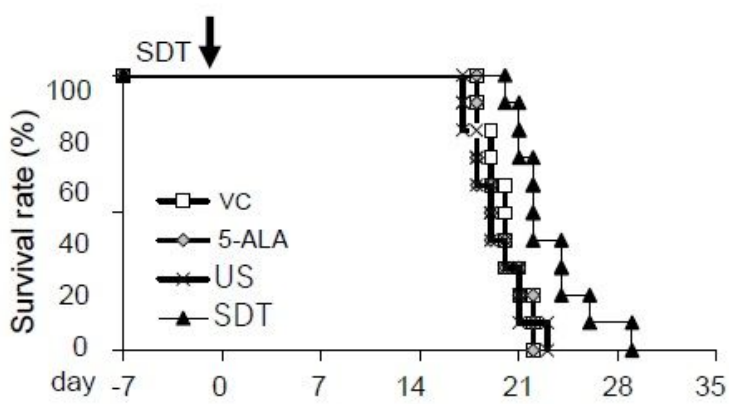

Figure 2

Anti-tumor effects of SDT in the mouse GSC-bearing glioma model. a. Protocol in mouse GSC-bearing glioma model; SDT performed $7 \mathrm{~d}$ after injection of GSCs. The mice were subjected to US $(1 \mathrm{MHz}, 2$ $\mathrm{W} / \mathrm{cm} 22 \mathrm{~min}$ ) at $3 \mathrm{~h}$ after injection of $200 \mathrm{mg} / \mathrm{kg} 5-\mathrm{ALA}$. Photographs indicate mice administered SDT, GSC injection area, and glioma. b. Representative DAB immunohistochemistry of apoptosis-related molecules on day 1 after SDT in glioma bearing mouse. Each specimen was co-stained with hematoxylin. c. GFP-labeled GSCs and tumor volume in brain on days 3-14 after SDT. Each column data indicates mean \pm SD. ${ }^{*} p<0.05$ by student's t-test. $d$. Changes in body weight before and after SDT e. Kaplan-Meier survival estimate (\%), no significant difference by log-rank test 
a.

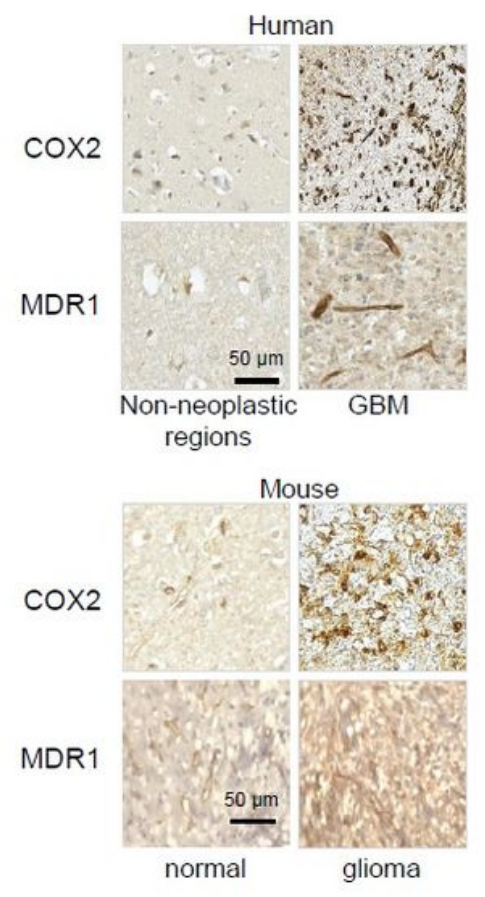

b.

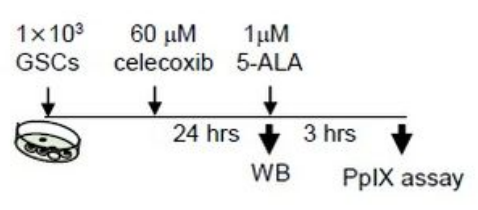

c.

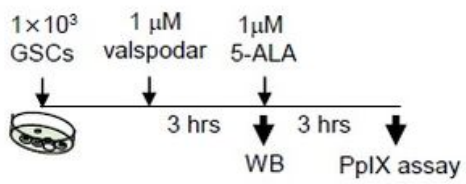

d.

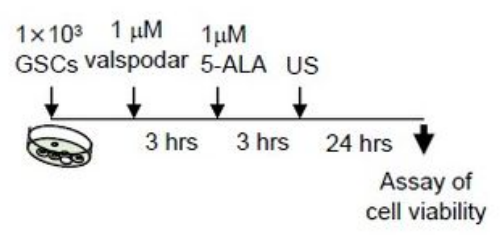

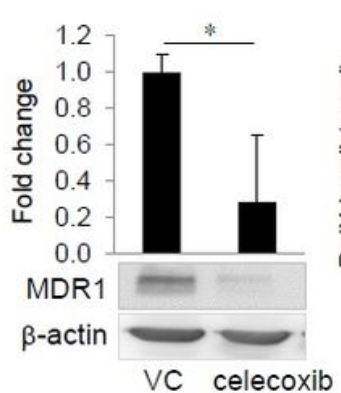
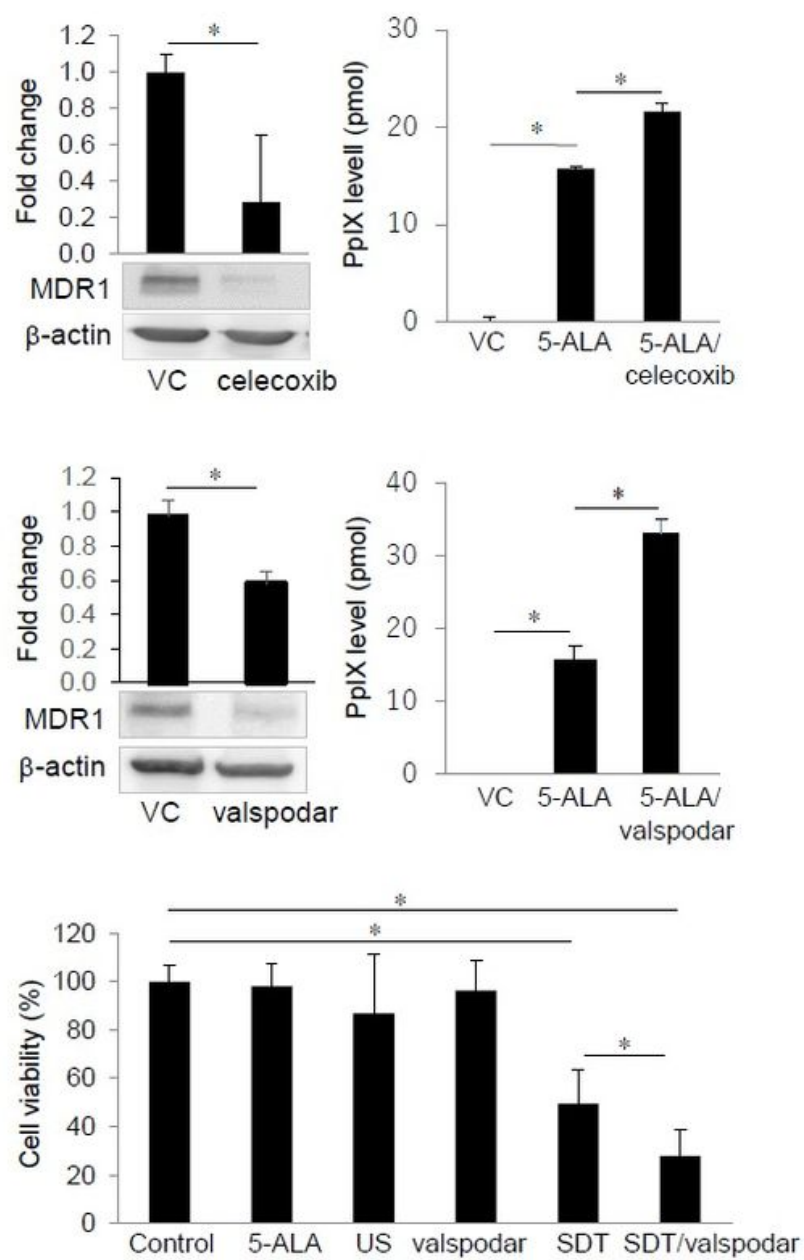

\section{Figure 3}

Elevation of PpXI through down-regulation of MDR1 leading to low cell viability in GSCs. a. Representative expression of COX-2 and MDR1 by DAB stain in human GBM and mice GSCs-bearing glioma model and each normal tissue. b. Changes in MDR1 expression and PpIX level treated with celecoxib and 5-ALA. WB analysis performed at $24 \mathrm{~h}$ after treatment with $60 \mathrm{uM}$ celecoxib and PpXI levels determined at $3 \mathrm{~h}$ after $1 \mathrm{uM}$ 5-ALA c. Changes in MDR expression and PpIX levels upon treatment with valspodar as a MDR1 inhibitor and 5-ALA. WB analysis performed at $3 \mathrm{~h}$ after valspodar and PpXI level determined at $3 \mathrm{~h}$ after 5-ALA. $\mathrm{d}$. Cell viability of GSCs treated with $1 \mathrm{uM} \mathrm{5-ALA}$ at $3 \mathrm{~h}$ after treatment with $1 \mathrm{uM}$ valspodar. US was performed at $3 \mathrm{~h}$ after 5-ALA. Each column data indicates mean \pm SD values $(n=8){ }^{*} p<0.05$ by student's t-test vs VC (2 groups) or Turkey-Kramer's test vs others (>3 groups) 
a.

In mouse

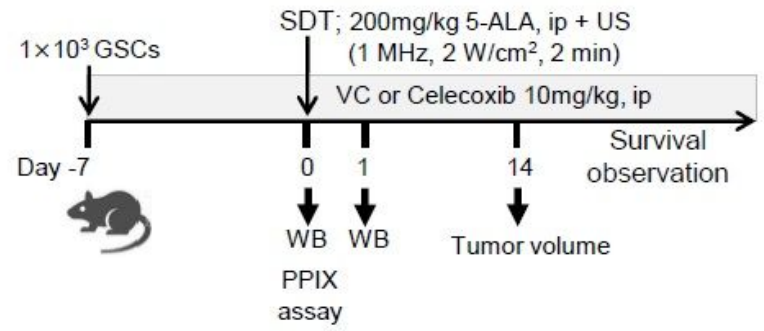

c.
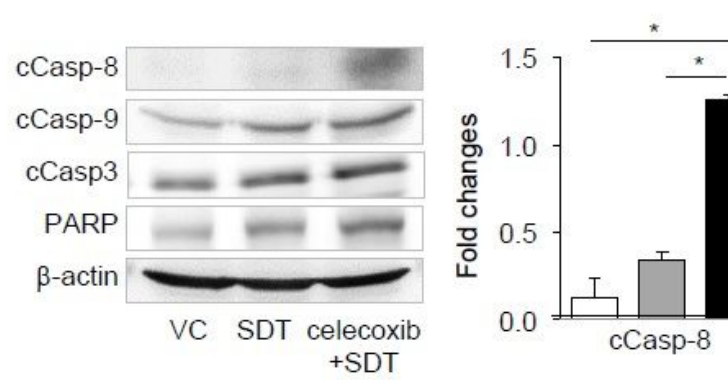

d.

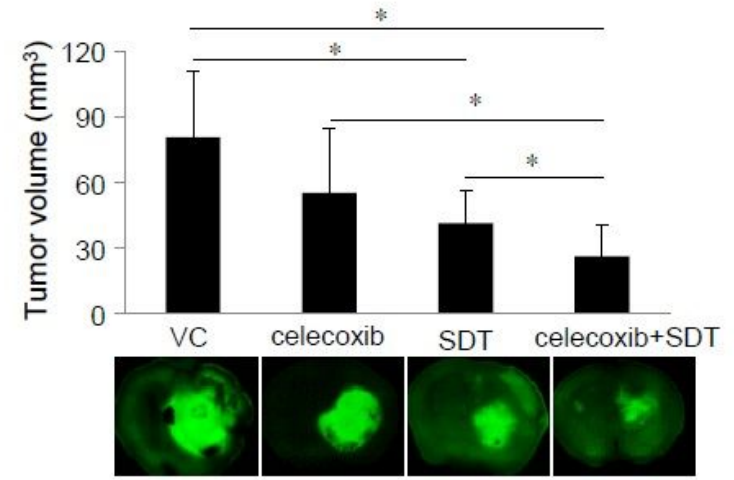

b.
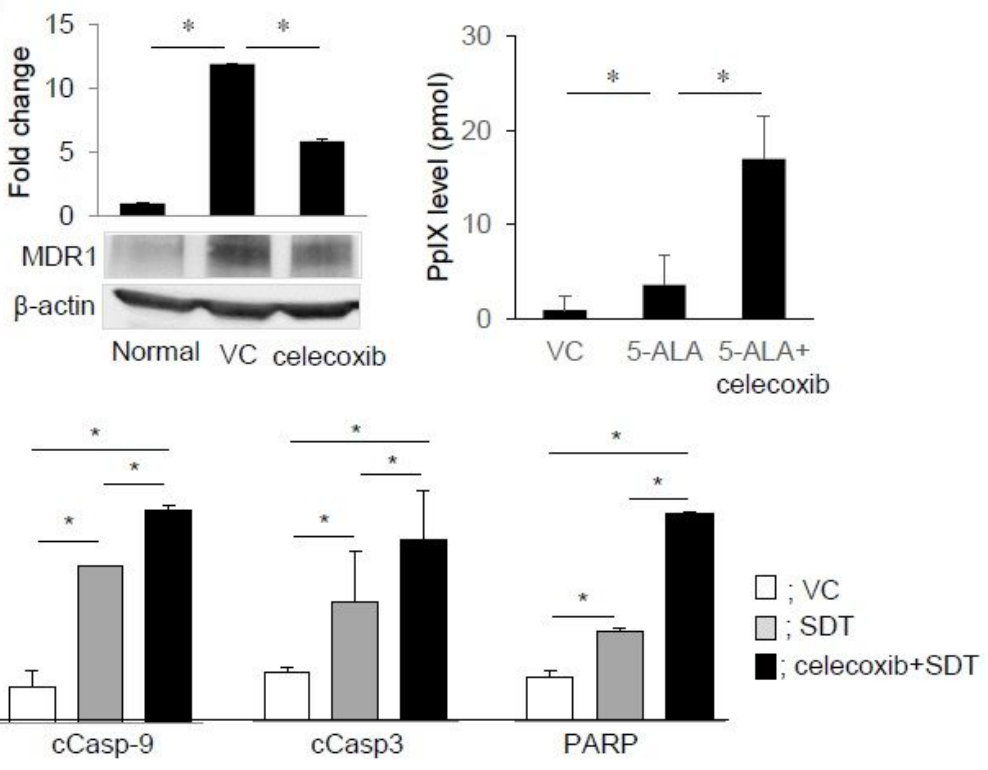

e.

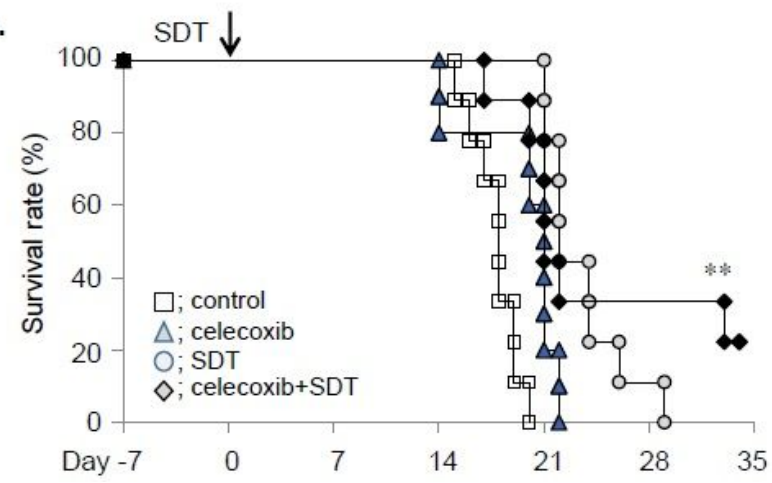

Figure 4

Elevation of cellular PpXI levels via MDR1 down-regulation by celecoxib augmented anti-tumor effects by SDT in the mouse glioma model. a. Protocol: celecoxib was continuously injected during the survival period after GSC injection. Seven days after GSCs injection, b. Expression of MDR1 by WB analysis and PpIX level at 3hr after SDT c. Expression of apoptosis-related molecules by WB at 1 day after SDT d. Tumor volume at $14 \mathrm{~d}$ after SDT. Each data indicates mean \pm SD $(n=6) .{ }^{*} p<0.05$ by Turkey-Kramer test. e. Kaplan-Meier survival estimate (\%) and survival period prolonged by the combination therapy with SDT and celecoxib. ${ }^{\star \star} \mathrm{p}<0.01$ vs other groups by the Log-rank test $(n=9-10)$ 
a.
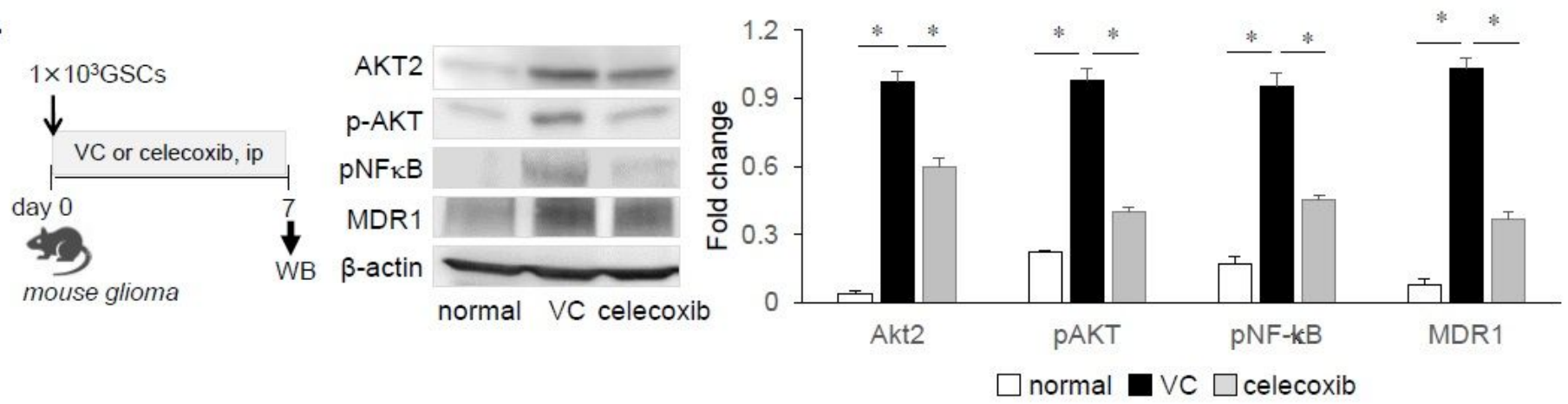

b.
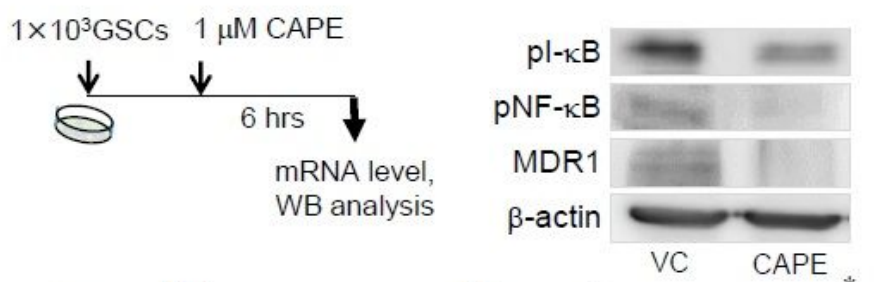

c.
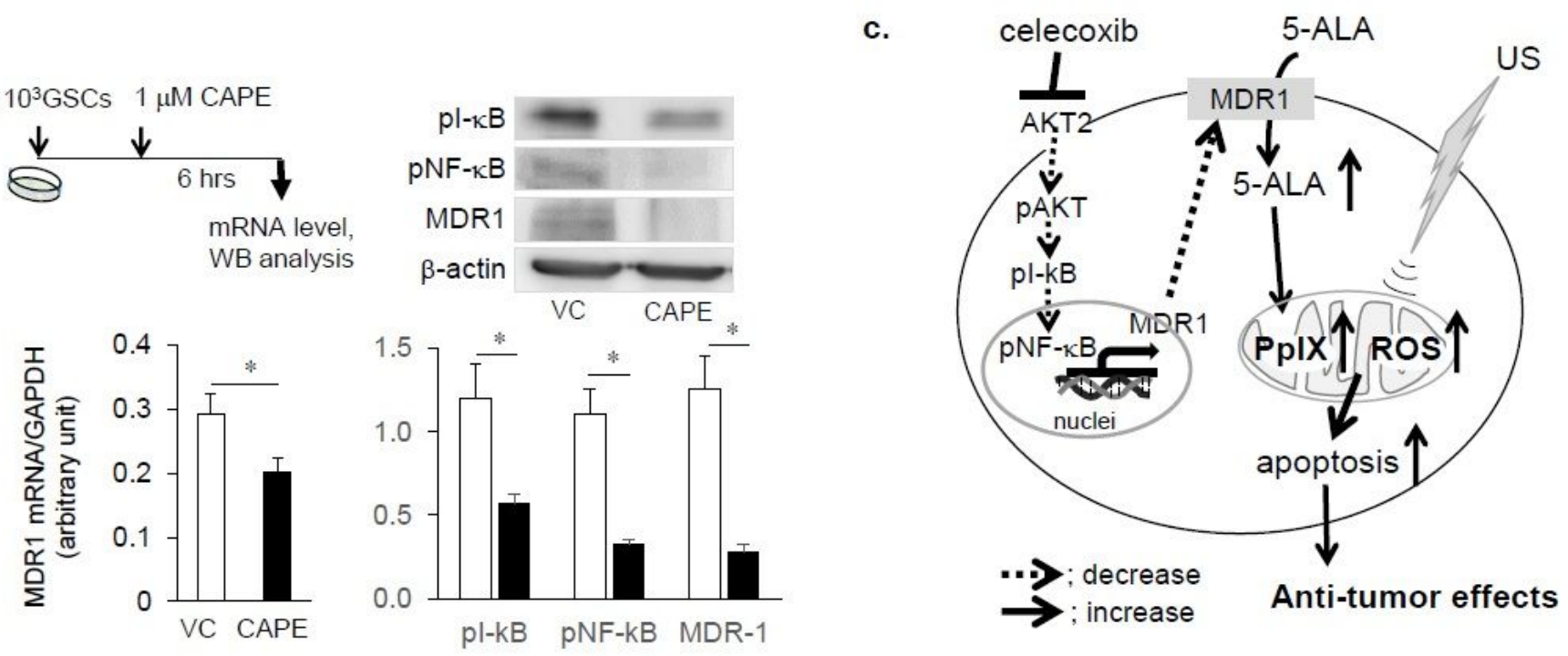

$\mathrm{VC}$

CAPE

\section{Figure 5}

Anti-tumor effects of SDT combined with the inhibition of the AKT/NF-KB/MDR1 pathway by celecoxib in the mouse GSCs-bearing glioma model. a. AKT/NF-KB signaling pathways and MDR1 expression upon western blotting after consecutive treatment with celecoxib for 7 days in the mouse GSC-bearing glioma model. b. Protein and mRNA levels of MDR1 in GSCs treated with or without $1 \mu \mathrm{M} \mathrm{CAPE}$, a potent and a specific inhibitor of NF-KB activation. c. Schematic representation of the anti-tumor effects upon combination therapy with SDT and celecoxib

\section{Supplementary Files}

This is a list of supplementary files associated with this preprint. Click to download.

- supplementFig.201203.pdf 